\title{
Calibration methods for rotating shadowband irradiometers and optimizing the calibration duration
}

\author{
Wilko Jessen ${ }^{1,2}$, Stefan Wilbert ${ }^{1}$, Bijan Nouri ${ }^{1}$, Norbert Geuder ${ }^{3}$, and Holger Fritz ${ }^{2}$ \\ ${ }^{1}$ German Aerospace Center, Institute of Solar Research, Qualification/Solar Energy Meteorology, \\ Plataforma Solar de Almería, Carretera de Senés s/n, km 5, 04200 Tabernas, Spain \\ ${ }^{2}$ Beuth University of Applied Sciences Berlin, Luxemburger Straße 10, 13353 Berlin, Germany \\ ${ }^{3}$ University of Applied Sciences Stuttgart, Schellingstraße 24, 70174 Stuttgart, Germany \\ Correspondence to: Stefan Wilbert (stefan.wilbert@dlr.de)
}

Received: 25 June 2015 - Published in Atmos. Meas. Tech. Discuss.: 6 October 2015

Revised: 20 March 2016 - Accepted: 29 March 2016 - Published: 12 April 2016

\begin{abstract}
Resource assessment for concentrated solar power (CSP) needs accurate direct normal irradiance (DNI) measurements. An option for such measurement campaigns is the use of thoroughly calibrated rotating shadowband irradiometers (RSIs). Calibration of RSIs and Si-sensors is complex because of the inhomogeneous spectral response of these sensors and incorporates the use of several correction functions. One calibration for a given atmospheric condition and air mass might not be suitable under different conditions. This paper covers procedures and requirements of two calibration methods for the calibration of rotating shadowband irradiometers. The necessary duration of acquisition of test measurements is examined with regard to the site-specific conditions at Plataforma Solar de Almería (PSA) in Spain. Seven data sets of long-term test measurements were collected. For each data set, calibration results of varying durations were compared to its respective long-term result. Our findings show that seasonal changes of environmental conditions are causing small but noticeable fluctuation of calibration results. Calibration results within certain periods (i.e. November to January and April to May) show a higher likelihood of deviation. These effects can partially be attenuated by including more measurements from outside these periods.

Consequently, the duration of calibrations at PSA can now be selected depending on the time of year in which measurements commence.
\end{abstract}

\section{Introduction}

Concentrated solar power (CSP) projects require accurate assessment of the available direct beam resource. Ground measurements have to be combined with satellite-derived data for this assessment. These ground measurements can be obtained by solar trackers and pyrheliometers or by rotating shadowband irradiometers (RSIs). Due to their lower susceptibility to soiling, RSIs have an important advantage over thermopile pyrheliometers during long-term deployment in remote and inaccessible sites, where daily cleaning of the instrument's aperture is not possible (Geuder and Quaschning, 2006; Pape et al., 2009; Maxwell et al., 1999). RSIs are also advantageous in terms of installation and maintenance costs and the typically achieved data availability.

RSIs consist of a pyranometer and a shadowband that rotates once per minute around the pyranometer such that the sensor is shaded for some time. Examples for RSIs can be seen in Fig. 1. When the shadowband is in its resting position, the global horizontal irradiance (GHI) is measured. Diffuse horizontal irradiance (DHI) is measured during the rotation and direct normal irradiance (DNI) is calculated using GHI, DHI and the solar zenith angle. RSIs are often called RSRs or RSPs, depending on the instrument manufacturer. Instead of irradiometer, radiometer or pyranometer appear in these names. The notation RSI refers to all instruments that measure irradiance by use of a rotating shadowband. There are two types of RSIs: RSIs with continuous and discontinuous rotation. The operational principal of RSIs with continuous rotation is explained in the following. At the beginning of 

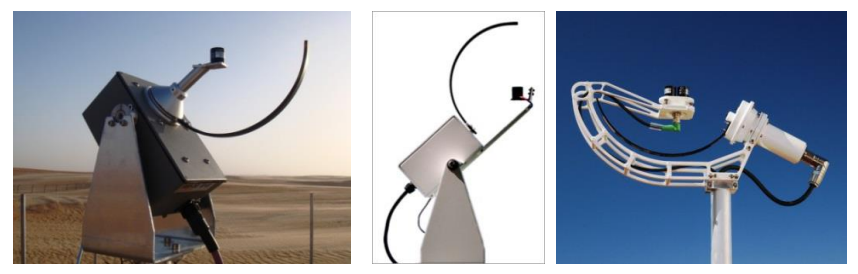

Figure 1. Rotating shadowband irradiometers. Left: RSR2 (irradiance Inc.), center: RSP4G (Reichert GmbH), right: Twin-RSI (CSP Services GmbH) (Wilbert et al., 2015).

the rotation, the shadowband is below the pyranometer, in its resting position. The rotation occurs with a constant angular velocity and takes approximately 1 to $2 \mathrm{~s}$. During the rotation, the irradiance is measured with a high and constant sampling rate (e.g. $1 \mathrm{kHz})$. This measurement is analyzed in order to derive GHI and DHI for the time of the rotation. In this paper only RSIs with continuous rotation of the shadowband are discussed. Such RSIs need a pyranometer with a fast response time ( $\ll 1 \mathrm{~ms}$, e.g. $10 \mu \mathrm{s})$. Thus, thermal sensors as described in ISO 9060 (1990) cannot be used. Instead, semiconductor sensors are used, e.g. the Si-pyranometer LI200SA (LI-COR, 2004).

RSIs with discontinuous rotation do not use a continuous and fast rotation, but a discontinuous stepwise rotation. Instead of measuring the complete signal during the rotation, only four points of it are measured (Harrison et al., 1994). First, the GHI is measured while the shadowband is in the resting position. Then the shadowband rotates from the resting position towards the position where it nearly shades the pyranometer, stops and a measurement is taken (e.g. for $1 \mathrm{~s}$ ). Then it continues rotating towards the position in which the shadow lies centered on the pyranometer and another measurement is taken. The last point is measured in a position in which the shadow just passed the pyranometer. RSIs with discontinuous rotation require a much more accurate adjustment of the instrument's azimuth orientation than RSIs with continuous rotation, as well as an exact time adjustment. Such RSIs will not be discussed here.

So far, RSIs with continuous rotation use the LI-COR LI-200SA pyranometer. This photodiode instrument experiences systematic errors due to cosine and temperature effects and its non-uniform spectral responsivity. A number of correction functions can be employed to reduce these errors significantly. The combination of the publications (King and Myers, 1997; King et al., 1998; Augustyn et al., 2004; Vignola, 2006) provide a set of functions which use the ambient temperature, solar zenith angle, air mass, GHI and DHI as input parameters. Geuder et al. (2008) introduced a separate set of correction functions which uses an additional spectral parameter determined from GHI, DHI, and DNI. An improved version of these corrections has been analyzed in Geuder et al. (2016).
A thorough calibration of RSIs with application of the correction functions is required for the utmost quality of measurements. The calibration procedures of thermopile pyranometers and pyrheliometers are well documented in standards such as ISO 9059 (1990), ISO 9846 (1993) and ISO 9847 (1992). These standards are not directly applicable to RSIs due to their inherent characteristics, especially because of the spectral selectivity of the Si-pyranometers used in RSIs. The inhomogeneous spectral response of results in the problem that the calibration for a given atmospheric condition and air mass might not work for a different condition with a corresponding different spectrum. Hence, specific calibration procedures for RSIs were developed by the German Aerospace Center (DLR), for example. In this paper DLR's calibration methods shall be called DLR2008 and VigKing (a detailed description follows in Sect. 2). They include significantly longer measuring periods and require measurements from a wider range of meteorological conditions than previously mentioned standards. The longer calibration durations ensure that the calibration is not derived only from extreme spectral conditions. In further deviation from the ISO standards for thermopile sensors, the RSI calibration methods by DLR assign more than one calibration factor for each instrument, since multiple components of solar irradiance with differing spectral composition and sensor responsivity are determined.

These RSI calibration methods have been applied at the Plataforma Solar de Almería (PSA) (latitude $37.0909^{\circ} \mathrm{N}$, longitude $-2.3581^{\circ} \mathrm{E}$, altitude $500 \mathrm{~m}$ a.m.s.l., semi-arid climate) for a number of years. However, some details are still under investigation to increase the reliability. A thorough assessment of the necessary calibration duration and seasonal influences on calibration results has now been carried out with several years of measurements from five RSIs. This paper outlines two RSI calibration procedures developed by DLR and presents the site-specific findings with regard to calibration durations and seasonal influences at PSA. It should be mentioned that only calibrations from one site (PSA) are evaluated and the conclusions are therefore partially site specific. However, although meteorological parameters may differ from site to site, the effect of fluctuating calibration results in accordance to seasonal influences is a general problem in outdoor calibration of Si-Pyranometers. Similar results are expected at sites of similar latitude and climate conditions. The site dependence of the performance is discussed in Geuder et al. (2016).

\section{Evaluated RSI calibration methods}

This paper discusses two calibration methods applied at PSA. In addition to the calibration, RSIs require the application of aforementioned correction functions in order to reduce the photodiode sensors' systematic error. Each of the calibration methods corresponds to a different set of correction 
Table 1. Parameter list.

\begin{tabular}{|c|c|c|}
\hline Symbol & Description & Units \\
\hline$\theta$ & temperature & ${ }^{\circ} \mathrm{C}$ \\
\hline$\Delta \mathrm{DNI}$ & conservative estimate of relative DNI uncertainty & $\%$ \\
\hline$\Delta$ Soil & uncertainty due to pyrheliometer soiling & $\%$ \\
\hline$\Pi$ & ratio of moving average to the long-term mean value & $\%$ \\
\hline$A$ & set of all timestamps of available data & date/hour/minute \\
\hline CFD & DHI calibration factor in DLR2008 calibration & - \\
\hline $\mathrm{CFd}$ & DHI calibration factor in VigKing calibration & - \\
\hline CFG & GHI calibration factor in DLR2008 calibration & - \\
\hline $\mathrm{CFg}$ & GHI calibration factor in VigKing calibration & - \\
\hline $\mathrm{CFn}$ & DNI calibration factor in VigKing calibration & - \\
\hline DHI & diffuse horizontal irradiance & $\mathrm{W} \mathrm{m}^{-2}$ \\
\hline DNI & direct normal irradiance & $\mathrm{W} \mathrm{m}^{-2}$ \\
\hline GHI & global horizontal irradiance & $\mathrm{W} \mathrm{m}^{-2}$ \\
\hline$L$ & long-term mean value of $R$ & - \\
\hline$M$ & moving average of $R$ & - \\
\hline$m$ & number of timestamps within one series of data & - \\
\hline$n$ & number of timestamps within a moving interval & - \\
\hline$R$ & $\begin{array}{l}\text { ratio of the reference irradiance to the corrected and calibrated } \\
\text { irradiance measured by the RSI }\end{array}$ & - \\
\hline SZA & solar zenith angle & $\circ$ \\
\hline$T$ & duration of the moving interval representing the calibration duration & days \\
\hline$t$ & timestamp for a $10 \mathrm{~min}$ interval & date/hour/minute \\
\hline
\end{tabular}

functions. Therefore, the following differentiates between the calibration method corresponding to functional corrections by Geuder et al. (2008; called DLR2008) and the calibration method corresponding to functional corrections by King, Myers, Augustyn and Vignola as published in King and Myers (1997), King et al. (1998), Augustyn et al. (2004), Vignola (2006) (called VigKing). Some functions in the latter set have been published in varying versions. The working set used in VigKing calibrations was summarized in Wilbert et al. (2015).

Both methods assign individual calibration factors to different irradiance components in addition to the LI-200SA Silicon-Pyranometer manufacturer's (LI-COR) calibration factor. The RSI is compared to a reference direct normal irradiance $\left(\mathrm{DNI}_{\mathrm{Ref}}\right)$ and a reference diffuse horizontal irradiance

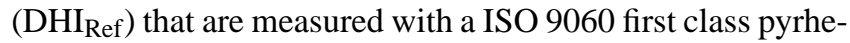
liometer and a secondary standard pyranometer (shaded with a shadowball), respectively. More specifically, Kipp\&Zonen $\mathrm{CH} 1$ or $\mathrm{CHP} 1$ pyrheliometers and CMP21 or CMP11 are used depending on the time interval under evaluation. The reference global horizontal irradiance $\left(\mathrm{GHI}_{\mathrm{Ref}}\right)$ is calculated via

$\mathrm{GHI}_{\mathrm{Ref}}=\mathrm{DNI}_{\mathrm{Ref}} \times \cos (\mathrm{SZA})+\mathrm{DHI}_{\mathrm{Ref}}$

from both reference instruments since this yields higher accuracy than GHI measurement by a pyranometer (see ISO 9060, 1990). Nonetheless, a second pyranometer that measures GHI directly is used to control the reference measurements as a redundancy check.
Table 2. Subscript list.

\begin{tabular}{ll}
\hline Subscript & Description \\
\hline acc & accepted data points \\
amb & ambient \\
cal & calibration \\
cor & functionally corrected RSI value \\
DHI & pertaining to DHI \\
DNI & pertaining to DNI \\
$d$ & at 12:00 pm \\
GHI & pertaining to GHI \\
max & maximum \\
Ref & reference for calibration \\
RSI & functionally corrected and calibrated RSI value \\
raw & uncorrected value measured by RSI \\
\hline
\end{tabular}

Data collection at PSA usually takes place continuously over the course of 30 to 120 days while the test RSI is positioned within less than $10 \mathrm{~m}$ from the reference instruments. Tables 1 and 2 provide the reader with a reference for parameters and subscripts used in equations. However, since RSIs are mostly employed in the context of CSP, only measurements under CSP operating conditions are included in the calculation of calibration factors. These conditions are specified later in detail. Due to the manifold environmental and operational influences which can occur during this time span, the raw data need to be screened and manually reviewed for errors and temporary system failures. The calculation of 
the calibration factors is based on minimizing the root mean square deviation (RMSD) but differs for both methods.

Another approach to RSI calibration was published in Kern (2010) which only compares GHI measurements from the photodiode to a reference and thus replaces the sensor manufacturer's calibration factor. However, this method is not subject of this paper.

\subsection{Calibration method DLR2008}

This calibration method assigns two calibration factors: CFG for GHI and CFD for DHI which are applied in accordance to Eqs. (2) to (4). Since both GHI and DHI have different spectral compositions, the sensor's responsivity differs among the two. The typical difference in responsivity is incorporated into the correction functions and hence the correction refers to the responsivity for the two components averaged over the group of instruments used for the development of the correction functions. However, the difference in responsivity for GHI and DHI is specific to each individual sensor as deviations of the spectral response between different instruments occur. Thus, better results are achieved by using separate calibration factors (Geuder et al., 2008). Because DNI is the desired measurand, CFG is optimized for determination of the DNI. The improved version presented in Geuder et al. (2016) allows a separate adjustment of calibration constants for $\mathrm{GHI}$ and DNI.

After calibration the final irradiance values measured by the RSI $\left(\mathrm{GHI}_{\mathrm{RSI}}, \mathrm{DHI}_{\mathrm{RSI}}\right.$ and $\left.\mathrm{DNI}_{\mathrm{RSI}}\right)$ are obtained as described in the following. $\mathrm{GHI}_{\mathrm{RSI}}$ is obtained by multiplying the calibration factor CFG to the functionally corrected global horizontal irradiance $\left(\mathrm{GHI}_{\mathrm{cor}}\right)$ :

$$
\mathrm{GHI}_{\mathrm{RSI}}=\mathrm{CFG} \times \mathrm{GHI}_{\mathrm{cor}} \text {. }
$$

The calculation of the functionally corrected and calibrated DHI differentiates between two cases. While the uncorrected DNI is at $2 \mathrm{~W} \mathrm{~m}^{-2}$ or above the equation

$$
\mathrm{DHI}_{\mathrm{RSI}}=\mathrm{CFD} \times \mathrm{DHI}_{\mathrm{cor}}
$$

is used. If the uncorrected DNI is lower than $2 \mathrm{~W} \mathrm{~m}^{-2}$, the calculation uses $\mathrm{GHI}$ and reads as

$\mathrm{DHI}_{\mathrm{RSI}}=\mathrm{CFD} \times \mathrm{GHI}_{\mathrm{cor}}$.

The reason is that at such low DHI values, usually there is little or no DNI, and thus DHI is equal to GHI; therefore the GHI value measured each second is more accurate than the DHI value derived from the measurement during the brief rotation.

The corrected and calibrated DNI, $\mathrm{DNI}_{\text {cor }}$ is determined from the corrected and calibrated $\mathrm{GHI}_{\mathrm{RSI}}, \mathrm{DHI}_{\mathrm{RSI}}$ and the solar zenith angle SZA as

$\mathrm{DNI}_{\mathrm{RSI}}=\frac{\mathrm{GHI}_{\mathrm{RSI}}-\mathrm{DHI}_{\mathrm{RSI}}}{\cos (\mathrm{SZA})}$.
The calibration process itself starts with data collection and documentation as follows. First, $\mathrm{GHI}_{\mathrm{Ref}}, \mathrm{DHI}_{\mathrm{Ref}}$ and $\mathrm{DNI}_{\mathrm{Ref}}$ are sampled every second and recorded as $1 \mathrm{~min}$ average values as well as the ambient pressure and temperature. Then the RSI values for GHI, DHI and DNI are averaged and recorded once per minute. The number of samples used per average value differs between the irradiance components since DHI can only be measured while the sensor is shaded (see sampling rates in Table 3). Furthermore, the sampling rate before calculation of 1 min values differs for RSR2 (irradiance Inc.), RSP-4G (Reichert GmbH) and Twin-RSI (CSP Services $\mathrm{GmbH}$ ). In RSP-4G and Twin-RSI sensors, the sensor temperature is also recorded. A comprehensive summary of how the irradiance components are derived from the photodiode's signal can be found in Wilbert et al. (2015).

The next step is the monitoring of the measurements. In order to identify and resolve operational problems, the recorded data of all instruments are scrutinized at least once per weekday by manually reviewing the reference and test data. Furthermore, the instruments are cleaned and inspected every weekday in situ for anomalies. The exact time of each cleaning event is documented. The redundant GHI measurement is used to control the operation of the reference instruments. Operational errors are documented in the calibration database. All relevant events concerning the measurement station and in the vicinity (e.g. construction works, maintenance of nearby instruments) are documented.

The data treatment includes the following steps. For each data channel 10 min mean values are calculated from the recorded $1 \mathrm{~min}$ averages: performing the calibration in 10 min time intervals reduces the signal deviation between reference and RSI at intermediate skies which results from the distance between the sensors and moving clouds. Then a screening algorithm performs a quality check of all recorded channels as recently presented in Geuder et al. (2015). Among other tests, the quality check tests and marks if measured values are physically possible, if their fluctuation (or lack of it) is realistic and if the data points have been manually flagged/commented during the measuring period. Furthermore, a soiling correction algorithm is applied to DNI Ref in accordance to the documented cleaning events following the method from Geuder and Quaschning (2006). Then, the LI-COR calibration factor $\mathrm{CF}_{\mathrm{Licor}}$ is applied to the RSI data.

Afterwards, the not yet calibrated and still uncorrected RSI time series, reference time series and time series of the signal deviations are checked for consistency by an expert. If one of the six irradiances seems to be unreliable, it is removed from the calibration data set. The manual discarding of data by expert intervention only excludes typically less than $1 \%$ of the calibration data set. In the improbable case that erroneous data are not excluded or that valid data are excluded, only small effects on the calibration results occur.

For RSI without temperature sensor (e.g. RSR2) the sensor temperature is estimated using the following Eq. (6) from Wilbert et al. (2015) based on GHI and ambient temperature 
Table 3. RSR2, RSP4G and Twin-RSI sampling rates (Wilbert et al., 2015).

\begin{tabular}{|c|c|c|c|c|}
\hline & Rotation frequency & GHI & DHI & DNI \\
\hline Twin RSI & $\begin{array}{l}1 /(30 \mathrm{~s}) \text { alternating for both } \\
\text { sensors }\end{array}$ & $1 / \mathrm{s}$ & $\begin{array}{l}\text { Shadowband correction } \\
\text { averaged with previous } \\
\text { value }\end{array}$ & $\begin{array}{l}\text { Calculated from GHI, DHI and solar } \\
\text { position as } 1 \text { min average with } \\
\text { correction for DHI drift }\end{array}$ \\
\hline RSR2 & $\begin{array}{l}\text { at least } 1 /(30 \mathrm{~s}) \text { up to } 1 /(5 \mathrm{~s}) \text { if } \\
20 \mathrm{~W} \mathrm{~m}^{-2} \text { change in } \mathrm{GHI}\end{array}$ & $1 /(5 \mathrm{~s})$ & Averaged for each rotation & Averaged for each rotation \\
\hline RSP4G & $1 /(60 s)$ & $1 / \mathrm{s}$ & $\begin{array}{l}\text { Calculated once per minute } \\
\text { as average of two rotations }\end{array}$ & $\begin{array}{l}\text { Calculated every second from } 1 \mathrm{~s} \text { GHI } \\
\text { samples. Averaged every } 60 \mathrm{~s}\end{array}$ \\
\hline
\end{tabular}

$\theta_{\mathrm{amb}}:$

$$
\begin{aligned}
\theta_{\mathrm{RSI}}= & \theta_{\mathrm{amb}}+\left(-4.883 \times 10^{-6} \times \mathrm{GHI}_{\text {raw }}^{2}+0.00953\right. \\
& \left.\times \mathrm{GHI}_{\text {raw }}-0.5\right) .
\end{aligned}
$$

Another estimation algorithm substitutes missing pressure measurements in all RSIs using the barometric formula. This is used in calculation of the apparent solar angle including refraction and is in particular required at low solar elevations.

Then the $\mathrm{GHI}_{\text {Ref }}$ is calculated by using the apparent sun height at the middle of each 10 min interval and the irradiance data are compared again. First, the deviation of $\mathrm{GHI}_{\text {raw }}$, $\mathrm{DHI}_{\text {raw }}$ and $\mathrm{DNI}_{\text {raw }}$ (RSI data with applied $\mathrm{CF}_{\text {Licor }}$ ) and the reference data are checked for plausibility by comparison against the reference irradiance components in scatterplots for each component. Implausible data are removed. Irradiance data which have been flagged by the screening algorithm as potentially erroneous are marked in the scatterplots and excluded. In a second check the deviation of RSI data from the reference data before and after application of the functional corrections (specific to the calibration method, here DLR2008) are compared to each other. Criteria for implausible data include high deviation between reference DNI (pyrheliometer) and calculated DNI from the reference pyranometers $\left(>8 \%\right.$ for $\mathrm{SZA}<75^{\circ}$; $>15 \%$ for greater SZAs) and high deviations between test and reference instruments (>25\%). Erroneous data are marked for exclusion and a comment is saved in the database.

The central step is the calculation of calibration factors. The solar elevation angle, $\mathrm{GHI}_{\text {Ref }}$ and $\mathrm{DHI}_{\text {Ref }}$ as well as their deviation from the functionally corrected but not yet calibrated RSI measurements $\mathrm{GHI}_{\text {cor }}$ and $\mathrm{DHI}_{\text {cor }}$ are filtered for their respective calibration limits (Table 4). The calibration limits define the acceptable range of irradiance and solar elevation angle for calibration as specified in Table 4 . Then the DHI calibration factor CFD is determined by minimization of the root mean square deviation (RMSD) of $\mathrm{DHI}_{\mathrm{RSI}}$ from $\mathrm{DHI}_{\text {Ref }}$ through variation of CFD. Thereafter, while ignoring the previous GHI and DHI data screening, the solar elevation angle, $\mathrm{DNI}_{\mathrm{Ref}}$, and its deviation from the corrected RSI measurement, $\mathrm{DNI}_{\text {cor }}$, is screened for its calibration limits (Table 4). With CFD calibration factor applied, the screened
Table 4. Calibration limits (Geuder et al., 2011).

\begin{tabular}{lr}
\hline Reference DNI $\left(\mathrm{W} \mathrm{m}^{-2}\right)$ & $>300$ \\
Reference GHI $\left(\mathrm{W} \mathrm{m}^{-2}\right)$ & $>10$ \\
Reference DHI $\left(\mathrm{W} \mathrm{m}^{-2}\right)$ & $>10$ \\
Solar elevation angle $\left(^{\circ}\right)$ & $>5$ \\
Max deviation of corrected RSI from reference $(\%)$ & \pm 25
\end{tabular}

data are used to determine the calibration factor CFG for GHI by minimization of the RMSD of $\mathrm{DNI}_{\mathrm{RSI}}$ from $\mathrm{DNI}_{\mathrm{Ref}}$ by variation of $\mathrm{CFG}$.

Finally, the calibration results are manually reviewed. The deviation of corrected RSI data from the reference before and after calibration is compared. Bias, standard deviation and RMSD of the corrected and calibrated RSI data from the reference are calculated and serve as indicators for the quality of the calibration. If further erroneous data are found, they can be marked for exclusion, and the calculation of the calibration constants is repeated.

The calibration procedure for the improved version (Geuder et al., 2016) is similar to the described one with the exception that CFG is optimized for GHI (instead of DNI). Here, two further calibration constants for DNI are introduced and fitted to the DNI dependent on its intensity after several filtering steps. This method, however, is not analyzed here.

\subsection{Calibration method VigKing}

VigKing determines three separate calibration factors $\mathrm{CFg}$, CFd and CFn for GHI, DHI and DNI respectively (Geuder et al., 2011). Each calibration factor is optimized for RMSD for the irradiance component it is applied to.

The calibration factors are applied in accordance to Eqs. (7) to (10). The functionally corrected and calibrated $\mathrm{GHI}\left(\mathrm{GHI}_{\mathrm{RSI}}\right)$ is obtained by multiplying the functionally corrected $\mathrm{GHI}_{\text {cor }}$ by the $\mathrm{GHI}$ calibration factor $\mathrm{CFg}$ :

$\mathrm{GHI}_{\mathrm{RSI}}=\mathrm{CFg} \times \mathrm{GHI}_{\mathrm{cor}}$.

The DHI correction is given with the functionally corrected $\mathrm{GHI}_{\text {cor }}$ as a parameter. After calibration, the functionally cor- 
rected and calibrated $\mathrm{DHI}_{\mathrm{RSI}}$ is calculated along the corrections from Vignola (2006) with $\mathrm{GHI}_{\mathrm{RSI}}$ as GHI input.

If $\mathrm{GHI}_{\mathrm{RSI}} \leq 865.2 \mathrm{~W} \mathrm{~m}^{-2}$, the DNI is calculated along Eq. (8) as

$$
\begin{aligned}
\mathrm{DHI}_{\mathrm{RSI}}= & \mathrm{CFd} \times\left[\mathrm{DHI}_{\mathrm{raw}}+\mathrm{GHI}_{\mathrm{RSI}} \times\left(-9.1 \times 10^{-11}\right.\right. \\
& \times \mathrm{GHI}_{\mathrm{RSI}^{3}}+2.3978 \times 10^{-7} \times \mathrm{GHI}_{\mathrm{RSI}}^{2} \\
& -2.31329234 \times 10^{-4} \times \mathrm{GHI}_{\mathrm{RSI}} \\
& +0.11067578794)]
\end{aligned}
$$

Equation (9) is used, if $\mathrm{GHI}_{\mathrm{RSI}}>865.2 \mathrm{~W} \mathrm{~m}^{-2}$ :

$$
\begin{aligned}
\mathrm{DHI}_{\mathrm{RSI}}= & \mathrm{CFd} \times\left[\mathrm{DHI}_{\mathrm{raw}}+\mathrm{GHI}_{\mathrm{RSI}} \times(0.0359-5.54\right. \\
& \left.\left.\times 10^{-6} \times \mathrm{GHI}_{\mathrm{RSI}}\right)\right] .
\end{aligned}
$$

The corrected and calibrated $\mathrm{DNI}_{\mathrm{RSI}}$ is determined with the DNI calibration factor $\mathrm{CFn}$ as

$\mathrm{DNI}_{\mathrm{RSI}}=\mathrm{CFn} \times \frac{\mathrm{GHI}_{\mathrm{RSI}}-\mathrm{DHI}_{\mathrm{RSI}}}{\cos (\mathrm{SZA})}$.

Note that the application of three calibration factors results in combinations of DNI, GHI and DHI that are not completely self-consistent. The calibration factor CFn is usually between 1.005 and 0.995 and hence the self-inconsistency is not very pronounced. The average of the absolute amount of $1-\mathrm{CFn}$ for 76 calibrations carried out at PSA between September 2013 and August 2015 is 0.0057.

In all aspects other than the correction functions and the assignment of a third calibration factor, the VigKing calibration method is identical to the method DLR2008 presented in Sect. 2.1. Contrary to the account given in Geuder et al. (2011) in today's practice, the same calibration limits (Table 4) are used in both calibration methods. The determination of the three calibration constants is done as follows.

$\mathrm{GHI}_{\text {Ref }}$ and $\mathrm{DHI}_{\text {Ref }}$ as well as their deviation from the corrected but not yet calibrated RSI measurements $\mathrm{GHI}_{\text {cor }}$ and $\mathrm{DHI}_{\text {cor }}$ are filtered for their respective calibration limits and solar elevation angle (Table 4). The screened data are then used to determine the GHI calibration factor CFg by minimization of the RMSD of the corrected and calibrated $\mathrm{GHI}_{\mathrm{RSI}}$ from $\mathrm{GHI}_{\text {Ref. }}$. Thereafter, the previous data screening for calibration limits is repeated with applied $\mathrm{CFg}$ before the screened data are used to determine the DHI calibration factor CFd by minimization of the RMSD of the corrected and calibrated $\mathrm{DHI}_{\mathrm{RSI}}$ from $\mathrm{DHI}_{\text {Ref }}$. Then, with applied $\mathrm{CFg}$ and $\mathrm{CFd}$, the corrected but not yet calibrated RSI measurement $\mathrm{DNI}_{\text {cor }}$ is screened for calibration limits (Table 4). Finally the screened data are used to determine the DNI calibration factors CFn by minimization of the RMSD of the corrected and calibrated $\mathrm{DNI}_{\mathrm{RSI}}$ from $\mathrm{DNI} \mathrm{Ref}_{\mathrm{Re}}$.

\section{Evaluation of RSI calibration duration and seasonal influences}

A first site-specific assessment of the necessary calibration duration at PSA has been presented in Geuder et al. (2014) in which a minimum measuring period of 30 days was recommended based on data collected from a single instrument. We investigated the subject further by use of a total of seven long-term data sets ranging from 251 to 1289 day duration collected over a period of 6.5 years from five RSI instruments (Table 5). Calibrating an instrument with the entire available long-term measuring period is considered the best achievable result.

Based on an application of moving averages the fluctuation of calibration results for different calibration durations was compared to the result of a long-term calibration over the whole period of available data. This was done separately for each of the seven long-term data sets. The deviation of calibration results from a long-term calibration with regard to DNI is represented by $\Pi_{\mathrm{DNI}}$ as defined in the following.

First the instrument is calibrated over the entire available long-term measuring period by either method from Sect. 2 . The thereby derived calibration factors are applied to the functionally corrected 10 min mean values of RSI measured irradiance from the calibration period. For the following steps the same manual and automatic data exclusions and the application of calibration limits (Table 4) as applied during the calibration process are kept in place. The remaining data are used to calculate the ratio $R_{\mathrm{DNI}}$ of reference to the calibrated RSI irradiance along

$R_{\mathrm{DNI}}(t)=\frac{\mathrm{DNI}_{\mathrm{Ref}}(t)}{\mathrm{DNI}_{\mathrm{RSI}}(t)}$.

The timestamp $t$ indicates the 10 min interval. Thereafter, the moving average (here: moving in steps of $24 \mathrm{~h}$ ) of $R_{\text {DNI }}$ is calculated as

$$
\begin{aligned}
& M_{\mathrm{DNI}}\left(T, t_{d}\right)=\frac{1}{n} \times \sum_{t} R_{\mathrm{DNI}}(t) \\
& \text { with } t \in\left[t_{d}-\frac{T}{2}, t_{d}+\frac{T}{2}\right] \bigcap A_{\mathrm{acc}},
\end{aligned}
$$

where $t_{d}$ represents a timestamp at noon and $T$ is the duration of the moving interval and thus represents the evaluated calibration duration. $T$ is an integer number of days. $A_{\text {acc }}$ is the set of all timestamps of the available data that were accepted after applying the exclusion parameters from Table 4 and the manual sort out by an expert. $n$ is the number of timestamps which are considered for the summation. As $t_{d}$ is always noon (UTC +1$)$ of a given day we calculate only one moving average $M_{\mathrm{DNI}}\left(T, t_{d}\right)$ for each day.

Additionally, $L_{\mathrm{DNI}}$ the mean of $R_{\mathrm{DNI}}$ over the entire measurement series is calculated along the equation

$$
L_{\mathrm{DNI}}=\frac{1}{m} \times \sum_{t} R_{\mathrm{DNI}}(t) \quad \text { with } \quad t \in A_{\mathrm{acc}}
$$


Table 5. Evaluated data sets.

\begin{tabular}{lllr}
\hline Instrument & From & To & $\begin{array}{r}\text { Duration } \\
\text { [days] }\end{array}$ \\
\hline RSP-4G-08-10-1 & 8 Aug 2008 & 16 Sep 2009 & 404 \\
RSP-4G-08-10-3 & 29 Jul 2010 & 7 Feb 2014 & 1289 \\
RSR2-0017 & 9 Sep 2009 & 16 May 2010 & 251 \\
RSR2-0018 & 17 May 2007 & 15 Oct 2008 & 517 \\
RSR2-0036 & 12 Nov 2007 & 12 Aug 2008 & 274 \\
RSR2-0039-1 & 12 Nov 2007 & 13 Aug 2008 & 292 \\
RSR2-0039-2 & 1 Jan 2011 & 18 Jan 2012 & 382 \\
\hline
\end{tabular}

where $m$ is the number of timestamps within the set $A_{\text {acc }}$. Finally,

$\Pi_{\mathrm{DNI}}\left(T, t_{d}\right)=\frac{M_{\mathrm{DNI}}\left(T, t_{d}\right)}{L_{\mathrm{DNI}}}$

is calculated as the ratio of the moving average $M_{\mathrm{DNI}}$ to the mean of $R_{\mathrm{DNI}}$ over the entire calibration period represented by $L_{\mathrm{DNI}}$. The evaluation method as described above was applied separately for both calibration methods DLR2008 and VigKing, and its findings are discussed in the following sections.

\subsection{Evaluation results for the method DLR2008}

Figure 2 displays the distribution of $\Pi_{\mathrm{DNI}}$ for each RSI data set (Table 5) and for varying calibration duration in form of box plots. In the type of box plots used in this paper the whiskers include $99.3 \%$ of all values in the case of normal distribution. The box itself includes $50 \%$ of all values with the first quartile below and the third quartile above its edges. The horizontal line signifies the median, while the circle symbolizes the arithmetic mean.

As expected, the whiskers of each data set get closer to zero with increased calibration duration $T$ since the influence of isolated extreme spectral conditions is evened out by the greater amount of data used. Similarly, the overall presence of outliers (exceptionally deviating calibration results) is reduced significantly. Due to the high volatility of calibration results for measuring periods below 14 days as seen in Fig. 2 such durations are considered as insufficient. In comparison to all other sensors, increasing the calibration duration from 1 to 30 days does not decrease the interquartile ranges significantly for the RSR2-0039-1. Since the power supply of the RSR2-0039 had to be exchanged between the measurement sets RSR2-0039-1 and RSR2-0039-2, we assume that this defect is the reason for the behavior.

Calibrations of 180 days duration or more on the other hand are too time-consuming in practice, especially considering the recommended frequency of recalibration of 2 years given in Geuder et al. (2014). Another disadvantage of very long-term calibrations is the lag time. Therefore, further analysis focuses on durations of 14, 30, 60, 90 and 120 days.

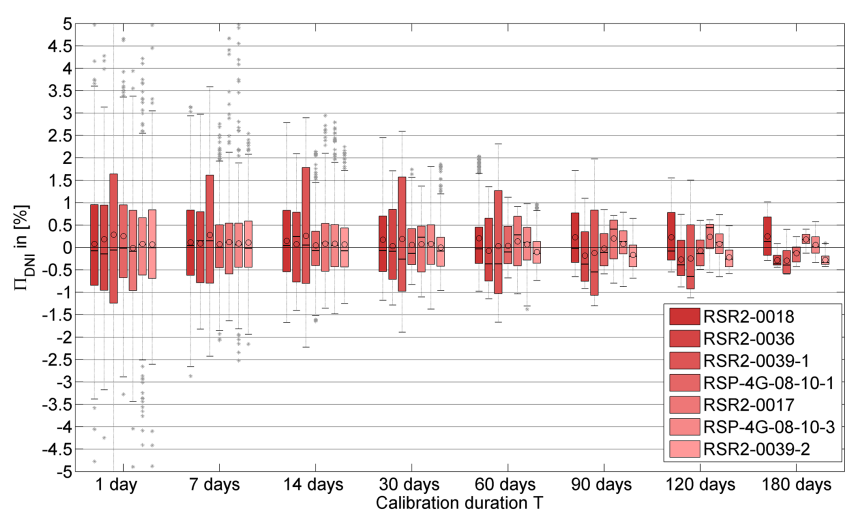

Figure 2. DLR2008: distribution of $\Pi_{\mathrm{DNI}}$ depending on calibration duration for seven data sets. $\Pi_{\mathrm{DNI}}$ represents the deviation of calibration results from a long-term calibration for DNI.

The $\Pi_{\mathrm{DNI}}$ were plotted over the time span of the longterm calibration measuring for one RSI data set at a time as shown in Fig. 3 for the RSP-4G-08-10-3 data set and calibration durations of $T=14$ days, $T=60$ days, $T=90$ days and $T=180$ days. The horizontal axis represents the date and the first day of each month is represented by vertical grid lines. The left vertical axis displays $\Pi_{\mathrm{DNI}}$ while the second vertical axis provides the daily number of usable timestamps. In this representation each plotted data point refers to the middle timestamp of its interval $t_{d}$.

The number of usable timestamps per day clearly coincides with the seasons. This is common to all data sets (Table 5) at hand and is a clear correlation to the daily daylight hours. Therefore, a calibration of the same duration differs in the amount of usable data dependent on the time of the year.

In order to derive recommendations for the necessary calibration duration for different times of the year, the $\Pi_{\mathrm{DNI}}$ of the seven data sets (Table 5) was sorted by the starting month of calibration and the combined distribution of all RSI data sets was visualized in box plots as presented in Fig. 4. This allows choosing the required duration in dependence of the starting time of measurements and the acceptable distribution of $\Pi_{\mathrm{DNI}}$. The same was done for $\Pi_{\mathrm{GHI}}$ and $\Pi_{\mathrm{DHI}}$ in Figs. 5 and 6. Since most of the time the greater part of GHI is due to DNI in CSP relevant regions, the seasonal course of $\Pi_{\mathrm{GHI}}$ (Fig. 5) distributions displays more similarities with the seasonal course of distributions of $\Pi_{\mathrm{DNI}}$ (Fig. 4) than of $\Pi_{\mathrm{DHI}}$ (Fig. 6). The highest volatility is observed in $\Pi_{\mathrm{DHI}}$ -

Most boxes in Fig. 4 are well centered on their respective arithmetic mean. The course of the $\Pi_{\mathrm{DNI}}$ reaches its maximum between November and January and its minimum between March and May. This indicates a small seasonal dependence of the calibration results. The longest calibration duration of $T=120$ days reaches its maximum $\Pi_{\mathrm{DNI}}$ in October, while a short duration of 14 days causes its respective maximum value to be 2 months later. This is due to the different calibration data set and the application of the start- 


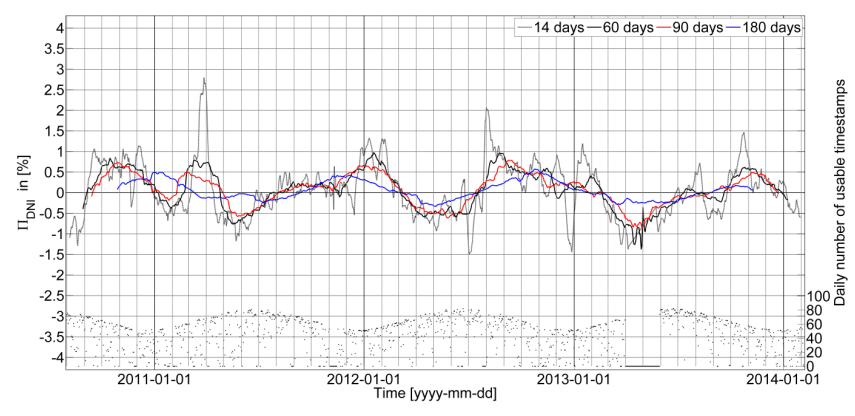

Figure 3. DLR2008: time series of $\Pi_{\mathrm{DNI}}$ for varying calibration duration for RSP-4G-08-10-3 with daily number of usable timestamps. $\Pi_{\mathrm{DNI}}$ represents the deviation of calibration results from a long-term calibration for DNI.

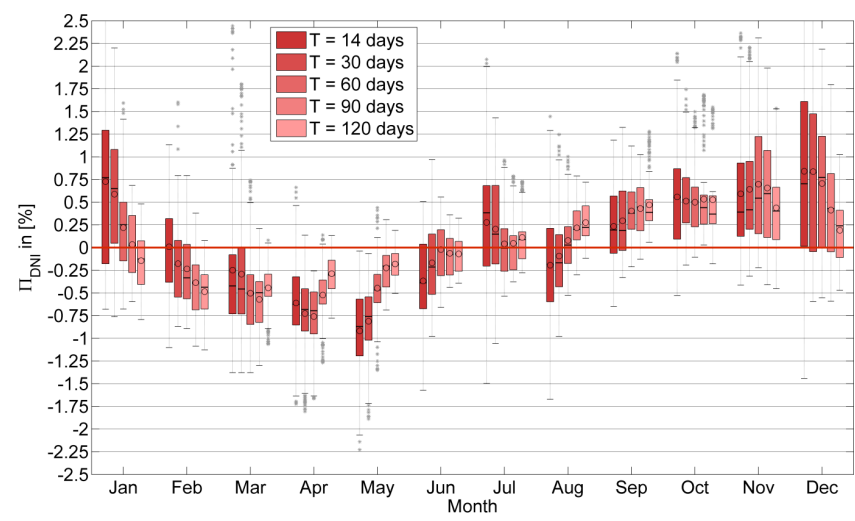

Figure 4. DLR2008: distribution of $\Pi_{\mathrm{DNI}}$ for $T=14$ days, 30 days, 60 days, 90 days and 120 days sorted by calibration starting month. $\Pi_{\mathrm{DNI}}$ represents the deviation of calibration results from a longterm calibration for DNI.

ing month for the $x$ axis. The general observation is that the longer the calibration duration, the closer the outcome will be to a long-term calibration. In this case, the conditions of the long-term calibration represent the application conditions, a long-term calibration is thus preferred. While this is true in most cases, some exceptions can be found. In Fig. 4 for starting dates in November and $T=60$ days the $\Pi_{\mathrm{DNI}}$ are distributed further from zero than with any other of the evaluated durations $T$. This is true for all statistical values that the box plot provides (whiskers, mean, etc.). However, a duration of $T=60$ days exhibits better distributions of $\Pi_{\mathrm{DNI}}$ than durations of 14 and 30 days for starting dates in the following month of December (better in terms of coincidence with $\Pi_{\mathrm{DNI}}=0$ ). For starting dates in January, the differences between the distribution of $\Pi_{\mathrm{DNI}}$ for 30 and 60 days duration is further increased. This indicates that the meteorological conditions in November and February are generally more suitable for DLR2008 calibration and later RSI application at PSA than in December and January.

Furthermore, starting dates in January produced closer coincidence between $\Pi_{\mathrm{DNI}}$ and zero with a duration of

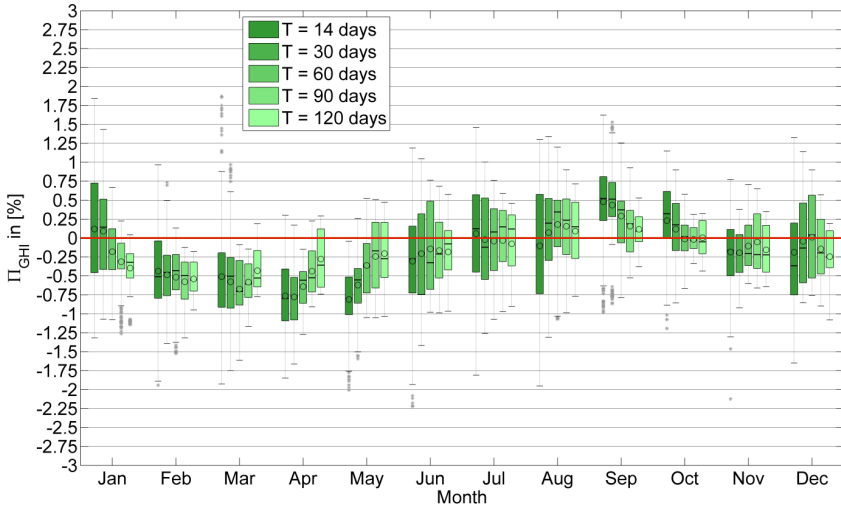

Figure 5. DLR2008: distribution of $\Pi_{\mathrm{GHI}}$ for $T=14$ days, 30 days, 60 days, 90 days and 120 days sorted by calibration starting month. $\Pi_{\mathrm{GHI}}$ represents the deviation of calibration results from a longterm calibration for GHI.

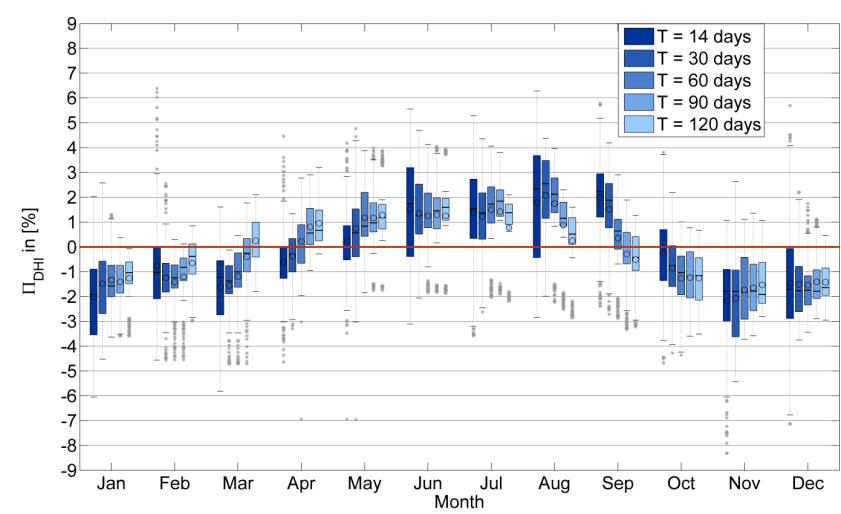

Figure 6. DLR2008: distribution of $\Pi_{\mathrm{DHI}}$ for $T=14$ days, 30 days, 60 days, 90 days and 120 days sorted by calibration starting month. $\Pi_{\mathrm{DHI}}$ represents the deviation of calibration results from a longterm calibration for DHI.

$T=90$ days than a duration of $T=120$ days. The $\Pi_{\mathrm{DNI}}$ of starting dates in February exhibited the closest coincidence with zero for durations of $T=60$ days along with $T=30$ days. 90 days periods starting in January and 60 days periods starting in February have in common that they end in April while the respective longer periods include all of April and a part of May. Remarkably, the duration $T=120$ days generated the greatest deviation of $\Pi_{\mathrm{DNI}}$ values from zero for starting dates in February. This is owed to the inclusion of the entire period of meteorological conditions in April and May.

However, out of all data visualized in Fig. 4 the $\Pi_{\text {DNI }}$ distributions for starting dates in May and June with $T=120$ days exhibited the smallest distance between upper and lower whiskers as well as the closest coincidence with $0 \%$ since the respective periods of time are dominated by the more suitable conditions from June onward. 
Table 6. DLR2008: Minimum required calibration duration in days for given maximum of $\Pi_{\mathrm{DNI}}$ and starting time.

\begin{tabular}{lrrrrrrrrrrrr}
\hline$\Pi_{\text {DNI,max }}$ & \multicolumn{10}{c}{ Month } \\
\hline & Jan & Feb & Mar & Apr & May & Jun & Jul & Aug & Sep & Oct & Nov & Dec \\
\hline $\pm 2.5 \%$ & 30 & 14 & 14 & 14 & 14 & 14 & 14 & 14 & 14 & 14 & 14 & 60 \\
$\pm 2.25 \%$ & 30 & 14 & 14 & 14 & 14 & 14 & 14 & 14 & 14 & 14 & 14 a & 60 \\
$\pm 2 \%$ & 60 & 14 & 14 & 14 & 30 & 14 & 14 & 14 & 14 & 14 & 90 & 90 \\
$\pm 1.5 \%$ & 60 & 14 & 14 & 90 & 60 & 30 & 30 & 30 & 14 & 30 & 120 & 120 \\
$\pm 1 \%$ & 90 & $30^{\mathrm{b}}$ & 120 & 90 & 60 & 30 & 60 & 30 & 90 & 90 & - & 120 \\
$\pm 0.75 \%$ & 90 & - & - & 120 & 90 & 90 & 90 & 120 & - & 90 & - & - \\
lowest & 90 & 30 & 120 & 120 & 120 & 120 & 120 & 120 & 120 & 120 & 120 & 120 \\
\hline
\end{tabular}

${ }^{\mathrm{a}} 60$ days is not suitable. ${ }^{\mathrm{b}}$ Only 60 days and 30 days are suitable.

As shown by these examples, the rough rule that longer calibration durations yield better results does not always apply, due to the meteorological conditions (i.e. spectral composition of irradiance) at the time. Exceptions to be considered are discussed in the following.

\subsection{Recommendations for DLR2008 calibration duration}

In consideration of the seasonal tendencies, it is recommendable to vary the calibration duration dependent on the month in which the measurements are commenced. This allows us to keep the monthly maximum deviation of $M_{\mathrm{DNI}}$ (Eq. 12) from $L_{\mathrm{DNI}}$ (Eq. 13) within a given maximum (hereafter called $\left.\Pi_{\mathrm{DNI}, \max }\right)$ and thus creates results of closer to constant viability while minimizing calibration duration. Table 6 provides a summary of required minimum durations for varying $\Pi_{\text {DNI,max }}$. Exceptions are marked in the table and explained in the caption. For example, even if a constant calibration duration of $T=60$ days is preferred to choosing the duration individually by month of the year, a reduction of the duration for calibrations starting in November to $T=30$ days only should be considered, since for this month a duration of $T=60$ days exhibited the highest $\Pi_{\mathrm{DNI}}$ in comparison to any other examined duration between 14 and 120 days.

The evaluation of seasonal influences was used to establish the correlation between $\Pi_{\mathrm{DNI}, \max }$, calibration duration and the month in which a calibration is commenced. Since $\Pi_{\text {DNI }}$ represents the deviation of individual short-term calibrations from the result of a long-term calibration, $\Pi_{\mathrm{DNI} \text {, max }}$ in combination with the relative standard uncertainty of the reference pyrheliometer $\left(\mathrm{DNI}_{\mathrm{Ref}}\right)$ can be used for a conservative estimate of calibration uncertainty:

$\Delta \mathrm{DNI}_{\mathrm{cal}} \approx \sqrt{\left(\Delta \mathrm{DNI}_{\mathrm{Ref}}\right)^{2}+(\Delta \mathrm{Soil})^{2}+\left(\Pi_{\mathrm{DNI}, \mathrm{max}}\right)^{2}}$.

In accordance with WMO (2010) the relative uncertainty of our reference pyrheliometer is $1.8 \%$ (95\% confidence level). We hence assume $\Delta \mathrm{DNI}_{\text {Ref }}=0.9 \%$ for the standard uncertainty. Additionally, an uncertainty due to pyrheliometer soiling of $\Delta$ Soil $\approx 0.2 \%$ can be estimated in respect of the find- ings in Geuder and Quaschning (2006). The calibration error can have a systematic component. The calibration error of the reference instruments causes a similar calibration error for the RSI calibration. The contribution of the soiling to the error is small due to the maintenance efforts at PSA and the soiling correction for the reference data. If less frequent cleaning is provided and no or insufficient soiling corrections are applied, additional systematic errors can occur, as the reference instruments are more sensitive to soiling than the RSIs under calibration.

An exemplary calculation for $\Pi_{\mathrm{DNI}, \max }=2.25 \%$ results in an estimated $\Delta \mathrm{DNI}_{\text {cal }} \approx 2.4 \%$ of the calibration uncertainty. It should be mentioned that this estimation includes the uncertainty caused by the spectral response of the solid state pyranometer that has to be expected for application at PSA after the calibration at PSA. Also other uncertainty contributions (e.g. directional, linearity and temperature effects) are partly included in this estimation because the bulk of the data in a calibration periods might belong to confined ranges of these parameters.

The calibration accuracy should also be related to the required accuracy for typical RSI applications. RSIs are usually applied for solar resource assessment that involves the combination of ground and satellite data. The uncertainty of the satellite data is typically much higher than the uncertainty of the ground measurements if best practices are followed for the measurements. An estimate of the maximal acceptable uncertainty of the combined data set from Meyer et al. (2008) is $4.5 \%$. According to this publication, the required accuracy can be reached with two satellite data sets of moderate quality and an uncertainty of $4 \%$ for the ground measurements. The achieved calibration uncertainty is therefore sufficient for the creation of these combined data sets and it is also sufficient for other applications (e.g. the validation of forecasted irradiance data; Schenk et al., 2015). 
Table 7. VigKing: Minimum required calibration duration in days for given maximum of $\Pi_{\mathrm{DNI}}$ and starting time.

\begin{tabular}{lrrrrrrrrrrrr}
\hline$\Pi_{\text {DNI,max }}$ & \multicolumn{10}{c}{ Month } \\
\hline & Jan & Feb & Mar & Apr & May & Jun & Jul & Aug & Sep & Oct & Nov & Dec \\
\hline $\pm 2.25 \%$ & 30 & 14 & 14 & 14 & 60 & 14 & 14 & 14 & 14 & 14 & 30 & 30 \\
$\pm 2 \%$ & 30 & 14 & 14 & 60 & 60 & 14 & 14 & 14 & 14 & 14 & 30 & 30 \\
$\pm 1.5 \%$ & 60 & 30 & 14 & 90 & 60 & 14 & 60 & 60 & 30 & 14 & 120 & 90 \\
$\pm 1 \%$ & 90 & $60^{\mathrm{a}}$ & $30^{\mathrm{a}}$ & 120 & 90 & 60 & 120 & 120 & 90 & - & - & 120 \\
$\pm 0.75 \%$ & $90^{\mathrm{a}}$ & - & - & 120 & 90 & 60 & - & - & 120 & - & - & - \\
lowest & 90 & 60 & 30 & 120 & 120 & $90^{\mathrm{b}}$ & 120 & 120 & 120 & 90 & 120 & 120 \\
\hline
\end{tabular}

a Only this duration. ${ }^{\mathrm{b}}$ Positioning of IQR better than 120 days.

\subsection{Recommendations for VigKing calibration duration}

The duration of VigKing calibrations is evaluated in the same fashion in the previous section for DLR2008.

In comparison to DLR2008, the VigKing method results in similar seasonal distributions of $\Pi_{\mathrm{DNI}}$. The rough rule that longer calibrations result in less deviation from the long-term calibration is also applicable in this case. However, some differences can be found. In Fig. 7 it is noticeable that the VigKing produces wider interquartile ranges of $\Pi_{\mathrm{DNI}}$. This is especially true for starting dates during the time from November to January. During this period, the distributions are exceptionally symmetrically centered around zero but exhibit the widest range of $\Pi_{\mathrm{DNI}}$ values.

With calibration method VigKing (Fig. 7) the $\Pi_{\mathrm{DNI}}$ distributions for starting dates in March were the closest to zero for durations of 30 days. Contrarily, in the following 2 months of April and May the distributions of $\Pi_{\mathrm{DNI}}$ for a duration of $T=30$ days deviate further from zero than for other durations. This observation indicates that the meteorological conditions during April and May are not well suited for VigKing calibrations. In the case of calibrations which start in April or May but reach well into the months of June or July a far closer coincidence of the $\Pi_{\mathrm{DNI}}$ with zero is achieved. This is caused by the more suitable conditions from June onward. A similar tendency was observed in DLR2008 (Fig. 4).

In DLR2008, the duration of 60 days produced the highest upper $\Pi_{\text {DNI }}$ whiskers among calibrations starting in November (Fig. 4). This is not true for VigKing (Fig. 7) where only $T=14$ days exhibits higher values than $T=60$ days. On the other hand, in regard to maximum $\Pi_{\mathrm{DNI}}$ and interquartile range a duration of $T=30$ days exhibits a more desirable distribution for this starting month than a duration of 60 days.

Measurements starting in October with 30 to 90 days duration resulted in smaller $\Pi_{\mathrm{DNI}}$ than 120 days duration due to the adverse conditions during the winter months. Considering the interquartile ranges of $\Pi_{\mathrm{DNI}}$, a duration of 90 days appears to perform best for calibrations starting in the month of October.

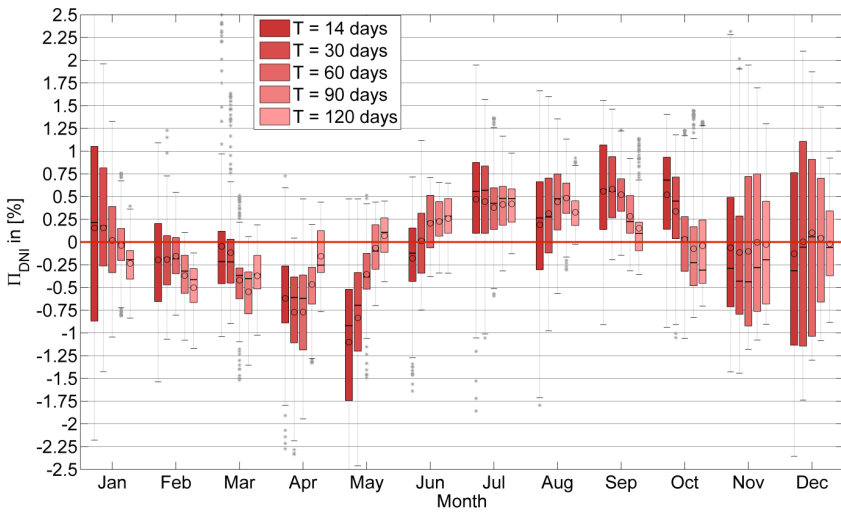

Figure 7. VigKing: distribution of $\Pi_{\mathrm{DNI}}$ for $T=14$ days, 30 days, 60 days, 90 days and 120 days sorted by calibration starting month for VigKing. $\Pi_{\mathrm{DNI}}$ represents the deviation of calibration results from a long-term calibration in regard to DNI.

Similarly as for DLR2008 calibrations, a table has been created to choose the calibration duration for VigKing depending on the month of the year and the desired $\Pi_{\text {DNI,max }}$ (Table 7).

If a constant calibration duration throughout the year is preferred, also for VigKing a duration of $T=60$ days is advised as a trade-off between producing results close to a longterm calibration and not consuming more time than reasonable. Similarly to DLR2008 one should resort to a shorter duration of $T=30$ days for calibrations starting in November. In VigKing the same is true for calibrations starting in March. Figure 7 suggests a deviation from a long-term calibration below $2 \%$ for $T=60$ days throughout the year.

\section{Conclusions}

The influence of the RSI calibration duration and the seasonal fluctuations of two calibration methods at PSA were investigated. Small but noticeable seasonal dependencies were observed. Also some fluctuations of RSI calibration results were found that are influenced by the calibration duration. 
Thus, it was possible to quantify relations which can be used to optimize the calibration duration dependent on the time of the year in which a calibration takes place.

Additionally, the findings allowed the identification of periods with higher likelihood of adverse meteorological conditions (November to January and April to May). Consequently, the duration of data acquisition for calibrations starting during these months should generally be longer than for calibrations starting during the rest of the year. In some cases it is advantageous to limit the duration of calibrations starting before these periods so that these periods are not used.

In order to apply the results of this analysis, two tables were constructed, which allows one to choose the calibration duration for both calibration methods depending on the month of the year in which measurements are commenced and the maximum tolerable value of $\Pi_{\mathrm{DNI}, \max }$, which represents the fluctuation of calibration results (Tables 6 and 7). For DLR2008 a constant calibration duration of 30 days throughout the year with the exception of calibrations starting in December (60 days) is sufficient to keep $\Pi_{\mathrm{DNI} \text {,max }}$ within $2.5 \%$. In VigKing calibrations, the same applies with the exception of using 60 days duration for calibrations starting in the month of May instead of December.

The subject of calibration uncertainty was briefly discussed in this paper and is a subject for further investigation. We found that during certain periods, deviation of calibration results exhibits positive (November to January) onesided tendencies and negative (April and May) one-sided tendencies. Further investigation is necessary to see if these seasonal effects can be attenuated by additional or improved functional corrections. In the future, the evaluation method used in this paper could also be applied to the improved version of DLR2008.

Acknowledgements. The RSR2 data sets were provided by Suntrace $\mathrm{GmbH}$ for which the authors wish to express their gratitude. DLR also thanks the EU for partially funding this research activity within the FP7 project SFERA2.

The article processing charges for this open-access

publication were covered by a Research

Centre of the Helmholtz Association.

Edited by: M. Hamilton

\section{References}

Augustyn, J., Geer, T., Stoffel, T., Kessler, R., Kern, E., Little, R., Vignola, F., and Boyson, B.: Update of Algorithm to Correct Direct Normal Irradiance Measurements Made with a Rotating Shadowband Pyranometer, in: Proc. Solar 2004, American Solar Energy Society, Boulder, USA, 2004.

Geuder, N. and Quaschning, V.: Soiling of irradiation sensors and method for soiling correction, Sol. Energy, 80, 1402-1409, 2006.
Geuder, N., Pulvermüller, B., and Vorbrugg, O.: Corrections for Rotating Shadowband Pyranometers for Solar Resource Assesment, Solar Energy + Applications, part of SPIE Optics + Photonics 2008, 10-14 August, San Diego, USA, 2008.

Geuder, N., Hanussek, M., Haller, J., Affolter, R., and Wilbert, S.: Comparison of Corrections and Calibration Procedures for Rotating Shadowband Irradiance Sensors, in: SolarPACES 2011, 20-23 September 2011, Granada, Spain, 2011.

Geuder, N., Affolter, R., Kraas, B., and Wilbert, S.: Long-term Behavior, Accuracy and Drift of LI-200 Pyranometers as Radiation Sensors in Rotating Shadowband Irradiometers (RSI), Energy Procedia, 49, 2330-2339, doi:10.1016/j.egypro.2014.03.247, 2014.

Geuder, N., Wolfertstetter, F., Wilbert, S., Schüler, D., Affolter, R., Kraas, B., Lüpfert, E., and Espinar, B.: Screening and Flagging of Solar Irradiation and Ancillary Meteorological Data, Energy Procedia, 69, 1989-1998, doi:10.1016/j.egypro.2015.03.205, 2015.

Geuder, N., Affolter, R., Goebel, O., Dahleh, B., Al Khawaja, M., Wilbert, S., Pape, B., and Pulvermueller, B.: Validation of Direct Beam Irradiance Measurements from Rotating Shadowband Pyranometers in a Region with Different Atmospheric Conditions, accepted, J. Sol. Energ., 2016.

Harrison, L., Michalsky, J., and Berndt, J.: Automated multifilter rotating shadow-band radiometer: an instrument for optical depth and radiation measurements, Appl. Optics, 33, 5118-5125, 1994.

ISO 9059: Solar energy - Calibration of field pyrheliometers by comparison to a reference pyrheliometer, International Organization for Standardization, Geneva, Switzerland, 1990.

ISO 9060: Solar energy - Specification and classification of instruments for measuring hemispherical solar and direct solar irradiation, International Organization for Standardization, Geneva, Switzerland, 1990.

ISO 9846: Solar energy - Calibration of a pyranometer using a pyrheliometer, International Organization for Standardization, Geneva, Switzerland, 1993.

ISO 9847: Solar energy - Calibration of field pyranometers by comparison to a reference pyranometer, International Organization for Standardization, Geneva, Switzerland, 1992.

Kern, E.: Calibration Methods for Silicon Photodiode Pyranometers used in Rotating Shadowband Radiometers, in: SolarPACES 2010, 21-24 September 2010, Perpignan, France, 2010.

King, D. L. and Myers, D. R.: Silicone Photodiode Pyranometers: Operational Characteristics, Historical Experiences and New Calibration Procedures, in: 26th IEEE Photovoltaic specialists Conference, 30 September-3 October 1997, Anaheim, California, USA, 1997.

King, D. L., Boyson, W. E., Hansen, B. R., and Bower, W. I.: Improved Accuracy for Low-Cost Solar Irradiance Sensors, in: 2nd World Conference and Exhibition on Photovoltaic Solar Energy Conversion Proceedings, 6-10 July 1998, Vienna, Austria, 1998.

LI-COR: Radiation measurement Instruments, Technical report, LICOR Biosciences, Lincoln, Nebraska, USA, 2004.

Maxwell, E. L., Wilcox, S. M., Cornwall, C., Marion, B., Alawaji, S. H., bin Mahfoodh, M., and Al-Amoudi, A.: Progress Report for Annex II - Assessment of Solar Radiation Resources in Saudi Arabia 1993-1997, NREL/TP-560-25374, National Renewable Energy Lab., Golden, Colorado, USA, 1999.

Meyer, R., Torres-Buron, J., Marquardt, G., Schwandt, M., Geuder, N., Hoyer-Klick, C., Lorenz, E., Hammer, A., and Beyer, H. G.: 
Combining solar irradiance measurements and various satellitederived products to a site-specific best estimate, SolarPACES Symposium, 4-7 March 2008, Las Vegas, USA, 2008.

Pape, B., Batlles, J., Geuder, N., Zurita Piñero, R., Adan, F., and Pulvermüller, B.: Soiling impact and correction formulas in solar measurements for csp projects, SolarPaces, Berlin, Germany, 2009.

Schenk, H., Hirsch, T., Wittmann, M., Wilbert, S., Keller, L., and Prahl, C.: Design and Operation of an Irradiance Measurement Network, Energy Procedia, 69, 2019-2030, doi:10.1016/j.egypro.2015.03.212, 2015.

Vignola, F.: Removing Systematic Errors from Rotating Shadowband Pyranometer Data, in: Solar 2006, American Solar Energy Society, 7-13 July 2006, Denver, Colorado, USA, 2006.
Wilbert, S., Geuder, N., Schwandt, M., Kraas, B., Jessen, W., Meyer, R., and Nouri, B.: Best Practices for Solar Irradiance Measurements with Rotating Shadowband Irradiometers, Technical Report IEA Task 46, Subtask B1 and INS project 1268, available at: http://task46.iea-shc.org/data/sites/1/publications/ INSRSI_IEA-Task46B1_BestPractices-RSI_150819.pdf (last access: 11 April 2016), 2015.

WMO: Guide to Meteorological Instruments and Methods of Observation, WMO-No. 8, 2010 Update, Seventh ed., World Meteorological Organization, Geneva, Switzerland, 2010. 